Check for updates

Cite this: Mater. Adv., 2020, 1,2395

Received 6th August 2020, Accepted 11th September 2020

DOI: $10.1039 / \mathrm{d} 0 \mathrm{ma00577k}$

rsc.li/materials-advances

\title{
Water-resistant 2D lead(II) iodide perovskites: correlation between optical properties and phase transitions $\dagger$
}

\author{
Bhushan P. Kore (D) and James M. Gardner (D) *
}

\begin{abstract}
The hybrid perovskite-based solar cells have achieved photovoltaic efficiencies comparable to that of the silicon-based solar cells; however, the light-absorbing perovskite materials are not stable and undergo rapid degradation in the presence of moisture. There are only a few water-stable 2D perovskite materials that have been explored so far. Keeping this in mind, we incorporated 3 different long-chain alkylammonium cations in 2D perovskites using a generic solution synthesis route where the saturated precursor solution was slowly cooled down to room temperature resulting in the single crystals of the $2 \mathrm{D}$ perovskites and studied their optical properties and stability against moisture. The prepared 2D perovskites demonstrated robust stability under ambient conditions as well as resistance to water. The main highlight of the present study is 2D perovskites emit bright green light in the 494-520 nm range even in the presence of water. We anticipate that our results on the water stable perovskite will not only motivate the use of these long alkyl chain cation-based 2D perovskite materials in perovskite solar cells for achieving the prolonged device stability but also for the next generation LEDs and display technologies.
\end{abstract}

\section{Introduction}

The last several years have witnessed a rapid surge in a new class of hybrid lead(II) halide perovskite-based solar cell technology. The hybrid perovskites possess excellent light-harvesting capability along with a high defect tolerance ${ }^{1-3}$ and long charge carrier diffusion lengths ${ }^{3-5}$ which are the physical properties of an ideal photovoltaic material. The hybrid perovskites based solar cells have surpassed PCE values over $25 \% ;^{6}$ however, the stability of hybrid perovskite under ambient conditions is still the most concerned area from a commercialization point of view of the technology. ${ }^{7,8}$

In the past, several attempts have been made to improve the stability of the $3 \mathrm{D}$ perovskites. The initial approach to improve the stability of the $3 \mathrm{D}$ perovskites was by preparing the mixed cation $^{9,10}$ and mixed halide compositions. ${ }^{11-13}$ The former approach has shown to improve the stability of the perovskite without compromising the band gap ${ }^{10,14}$ whereas the later approach altered the band gap of the perovskite ${ }^{11,15}$ and when devices made of mixed halide composition (I:Br) were kept under continuous light illumination for long enough time they

Department of Chemistry, Division of Applied Physical Chemistry, KTH Royal Institute of Technology, SE-100 44 Stockholm, Sweden. E-mail: jgardner@kth.se $\dagger$ Electronic supplementary information (ESI) available: Experimental details, characterization tools, XRD data of thin films and powder samples, absorption and PL emission spectra of 2D perovskites, PL QY results, photographs of 2D perovskites under UV-light illumination. See DOI: 10.1039/d0ma00577k phase segregated into iodide and bromide rich regions. ${ }^{16-18}$ Even though these perovskites show improved device performance with moderately improved stability, but when the devices come in contact with moisture the impurity phase of the $\mathrm{PbI}_{2}{ }^{19}$ and other non-photoactive/non-perovskite phases appear ${ }^{20}$ and gradually increase with time. Therefore, it is important to encapsulate the light-absorbing perovskite with moisture-resistant material to prevent the degradation of perovskite from moisture.

In $2 \mathrm{D}$ layered perovskites, a bulky organic cation makes the 2D perovskite hydrophobic leading to the higher device stability against moisture. ${ }^{21-23}$ Furthermore, the stacking of organicinorganic layers leads to the natural quantum well structure, where the inorganic layers serve as the potential wells while the organic layers act as potential barriers. ${ }^{24,25}$ Another interesting feature of $2 \mathrm{D}$ perovskites is that the dielectric and excitonic properties related to this quantum well structure can be easily tuned by alloying halide anions and varying the thickness of the inorganic layer. $^{25-27}$

Coating 2D layer on the top of 3D perovskite is one of the most commonly used approach these days for preventing the $3 \mathrm{D}$ perovskite from the moisture. ${ }^{28-30}$ There are several 2D perovskite materials have been explored using this approach and significant improvement in the device stability was achieved but very few of them showed higher photovoltaic efficiencies. ${ }^{2,29-31}$

From the commercialization of perovskite-based solar cell technology point of view, there is an urgent need to improve the stability of the perovskites in the near future. In literature, 
there are very few $2 \mathrm{D}$ perovskite materials reported that are stable under humid conditions, ${ }^{29,32,33}$ thermal stress ${ }^{34-36}$ and continuous illumination to UV light. ${ }^{29}$ The quest to develop a stable perovskite material that can outperform under ambient conditions has motivated us to investigate the long carbon chain $\left(\mathrm{C}_{14}, \mathrm{C}_{16}\right.$ and $\left.\mathrm{C}_{18}\right)$ organic cations in $2 \mathrm{D}$ perovskite.

In this study, we synthesized alkylammonium based $\left(\mathrm{C}_{n} \mathrm{H}_{2 n+1}-\right.$ $\left.\mathrm{NH}_{3}\right)_{2} \mathrm{PbI}_{4} 2 \mathrm{D}$ perovskite systems. The desired compounds were obtained by employing a simple slow precipitation method. The stability of the prepared compounds was tested under ambient conditions as well as by immersing them in water. The bulk compounds do not show any degradation or impurity phases when exposed to water for hours to days. Due to their water resistance these materials could be interesting candidates for use as a passivating layer for $3 \mathrm{D}$ perovskites to protect against moisture and to improve the longevity of the $3 \mathrm{D}$ perovskite photovoltaic devices.

\section{Results and discussion}

The two-dimensional hybrids with a general formula $\left(\mathrm{C}_{n} \mathrm{H}_{2 n+1}\right.$ $\left.\mathrm{NH}_{3}\right)_{2} \mathrm{MX}_{4}$, where $\mathrm{M}$ is a metal cation ( $\mathrm{Pb}, \mathrm{Cd}, \mathrm{Cu}, \mathrm{Mn}$ ) and $\mathrm{X}$ is halogen $(\mathrm{Cl}, \mathrm{Br}, \mathrm{I})$ have been studied in past for diversity in their structure since they show structural phase transitions below and near the room temperature depending upon the length and conformation of the alkylammonium chains. ${ }^{37-40}$

The general structure of the lead-based $\left(\mathrm{C}_{n} \mathrm{H}_{2 n+1} \mathrm{NH}_{3}\right)_{2} \mathrm{PbI}_{4}$ $(n=14,16$ and 18) 2D perovskites consist of alternating layers of a corner shared $\mathrm{PbI}_{6}$ octahedra and alkylammonium chains, the schematic of the crystal structures of $n=14$ compound is shown in Fig. 1a and b. The alkylammonium chains in the organic layers are connected to the inorganic layer by charge assisted $(\mathrm{N}-\mathrm{H} \cdots \mathrm{I})$ hydrogen bonds. ${ }^{37,41}$ The inorganic layer consists of a corner sharing $\mathrm{PbI}_{6}$ octahedra. Barman et al., were the first to propose the crystal structure of these perovskites based on the powder X-ray diffraction (XRD) and some other spectroscopic measurements, where they propose a non-interdigitated arrangement of the alkylammonium chains with two possible

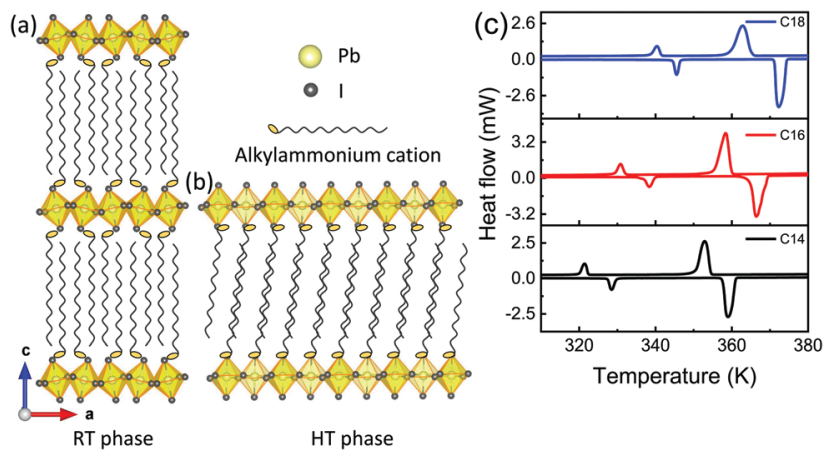

Fig. 1 Schematic of the crystal structure of the $\left(\mathrm{C}_{14} \mathrm{H}_{29} \mathrm{NH}_{3}\right)_{2} \mathrm{Pbl}_{4}$ perovskite (a) at room temperature (RT) and (b) at high temperature $(\mathrm{HT})$ with interdigitated arrangement of alkylammonium chains. (c) Differential scanning calorimetry (DSC) curves of the $\left(\mathrm{C}_{n} \mathrm{H}_{2 n+1} \mathrm{NH}_{3}\right)_{2} \mathrm{Pbl}_{4}(n=14,16$ and 18) 2D perovskite powder samples measured in both heating and cooling cycle. orientations. $^{42}$ Later Billing et al., did temperature-dependent single crystal XRD measurements and revealed that the alkylammonium chains are actually ordered with an interdigitated arrangement and bound by van der Waals forces. ${ }^{41}$ These $2 \mathrm{D}$ materials undergo first-order phase transitions and the phase transitions are mainly attributed to the thermal motion of the alkylammonium chains relative to the inorganic layers and rotation of the inorganic planes relative to one another. ${ }^{41,42}$ The chains are more disordered in the HT phase. The first phase transition refers to pre-melting and the second phase transition refers to the melting of alkylammonium chains. ${ }^{41}$

The phase transitions in these compounds were also under conflict. Billing et al., ${ }^{41}$ observed a reversible phase transition whereas Barman et al., ${ }^{42}$ observed pre-melting transition when the samples were kept for some days and then repeating the calorimetric study. The conflict in the crystal structure could be associated with the reversibility or irreversibility of the phase transitions. We carried out the DSC measurements on the powder samples of 2D perovskites and observed almost completely reversible phase transitions, as shown in Fig. 1c. Furthermore, the DSC results show the presence of thermal hysteresis as the exotherms related to the pre-melting and melting transitions occur at lower temperatures compared to the corresponding endotherms. The phase after the melting transition is not discussed in the literature due to the loss of the crystallinity of the crystals.

In these $2 \mathrm{D}$ perovskites, during the first phase transition the crystal system change from the orthorhombic to the monoclinic unit cell and the unit cell dimension get halved after the phase transition. ${ }^{41}$ The unit cell of these $2 \mathrm{D}$ materials at RT consist of two organic layers sandwiched between 3 inorganic layers (Fig. 1a). After the first phase transition, the structure of the 2D perovskite changes to the monoclinic unit cell in which a single layer of organic cations is sandwiched between the two inorganic layers as shown in Fig. 1b. In the RT phase, the lead atoms from the two consecutive inorganic layers are offset, and this leads to the staggered arrangement of the adjacent layers (Fig. 1a), whereas in the monoclinic structure they are well aligned from one layer to another, resulting in the overlapped arrangement of the adjacent layers.

Fig. 2a shows the absorption and PL emission spectra of thin film of $(n=14) 2 \mathrm{D}$ perovskite. The absorption spectra show an absorption maximum at $488 \mathrm{~nm}$ and a second peak at longer wavelengths for all of the three compounds (Fig. S1a and b, ESI $\dagger$ ). The PL emission spectra also reveal the presence of two unresolved peaks in all three samples, which is complimentary to the absorption results. The presence of two unresolved peaks in absorption and PL emission spectra could originate from the simultaneous coexistence of the two crystallographic phases in these compounds.

These 2D perovskite compounds undergo phase transitions that occur slightly above the RT. In this study, the thin-films of these compounds were prepared by spin coating technique and were annealed at $100{ }^{\circ} \mathrm{C}$. This annealing temperature is large enough to cross the phase transitions. The presence of shoulder peak in the PL spectra suggests the presence of both the RT phase and HT phase in the annealed films. The longer wavelength peak 

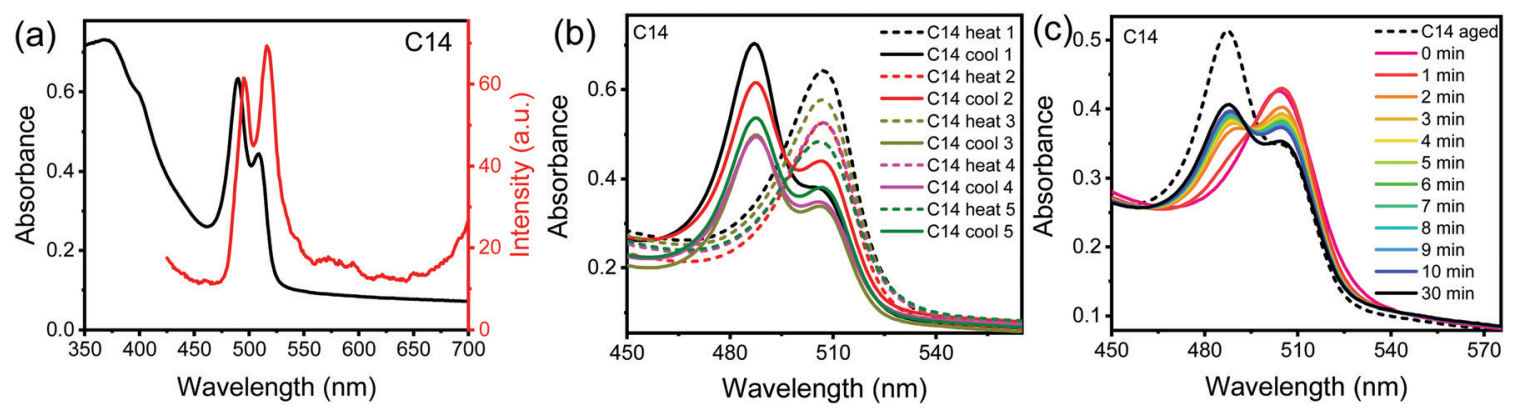

Fig. 2 (a) Absorption and photoluminescence spectra of $\left(\mathrm{C}_{14} \mathrm{H}_{29} \mathrm{NH}_{3}\right)_{2} \mathrm{Pbl}_{4} 2 \mathrm{D}$ perovskite thin films. (b) Absorption spectra of $\left(\mathrm{C}_{14} \mathrm{H}_{29} \mathrm{NH}_{3}\right)_{2} \mathrm{Pbl}{ }_{4} 2 \mathrm{D}$ perovskite thin film recorded just after annealing the thin film at $100{ }^{\circ} \mathrm{C}$ (dotted curve) and thin film cooled to room temperature for $1 \mathrm{~h}$ (solid curve). (c) Evolution of absorption spectra of $\left(\mathrm{C}_{14} \mathrm{H}_{29} \mathrm{NH}_{3}\right)_{2} \mathrm{Pbl}_{4} 2 \mathrm{D}$ perovskite thin films just after annealing at $100{ }^{\circ} \mathrm{C}$ with time.

originates from the presence of HT phase, because through the annealing process $\left(100^{\circ} \mathrm{C}\right)$ of the samples, a change in color from yellow to orange is observed. The change in color is precisely what would be expected from the HT phase having a redshifted PL emission. From the XRD results it is difficult to observe the presence of the HT phase since the XRD patterns of the RT phase and HT phase are similar and as a thin-film the materials are preferentially oriented such that only certain diffraction peaks are observed compared to the isotropically oriented powder samples (Fig. S2, ESI $\dagger$ ).

To further confirm the origin of the peak at longer wavelength region, we compared the PL emission spectra of the freshly prepared powder samples, the powder samples were not annealed at all, with that of the thin films. The PL emission peak at a shorter wavelength exactly overlaps with the PL emission spectra recorded in the powder samples (Fig. S3, ESI $\dagger$ ), indicating that the peak at longer wavelengths results from the presence of the HT phase.

To rule out the possibility of emission at the longer wavelengths due to defect related states, we carried out absorption measurements after several cycles of annealing the samples above the phase transition temperature and cooling down to room temperature. The absorption spectra of all the three $2 \mathrm{D}$ perovskite samples (Fig. 2b and Fig. S4a, b, ESI $\dagger$ ) show emergence of absorption peak at longer wavelength after annealing the samples, which corresponds to the high temperature phase that is suppressed when the samples cools down to room temperature. The reversibility of this phase transitions can be observed even after several cycles of heating and cooling, which clearly illustrates that the longer wavelength absorption peak does not correspond to the defect related states but due the presence of high temperature phase. Furthermore, the time evolution of absorption spectra (Fig. 2c and Fig. S4c, d, ESI $\dagger$ ) just after annealing the thin films demonstrates how a gradual transformation from HT phase to RT phase take place in these 2D perovskites. In these figures an isobestic point is present at $495 \mathrm{~nm}$, indicating that the materials transition seemlessly from one phase to another.

We speculate that the observed reversibility and irreversibility of phase transitions in powder and thin films of the 2D perovskites is due to the fact that in a thin film the crystals of the 2D perovskites have no place to move as they relax to the RT phase and as a result are trapped. In a powder there is no such guarantee that crystals are in direct contact with one another and the inorganic planes are freer to rotate.

All three of the 2D lead iodide perovskite compounds exhibit intense green light emission upon UV light exposure (Fig. S5, ESI $\dagger$ ). We measured absolute PL QYs using integrating sphere and found to be 2,2 and $3 \%$ for $n=14,16$ and 182 D perovskite powder samples, respectively. We further verified the PL QY results of 2D perovskite thin film samples by comparing the PL emission of 2D perovskite with a standard dye of known PL QY (see Fig. S6, ESI $\dagger$ ). The PL QY values estimated from relative PL QY measurements are 10,12 and 15\% for $n=14,16$ and 18 perovskites. Such a large variation in PL QY values using two different techniques could be due to the difference in the light detection technique in relative and absolute PL QY measurements and variation in the quality (grain size, defects, crystallinity of the sample) of the samples. The reason for lower PL QYs in powder samples of 2D perovskites could be more self absorption losses inside the sample. The differences in these two measurements and their data are further expanded upon in the ESI $\dagger$ section on absolute and relative PL QY.

The absorption spectra of $n=14,16$ and 18 samples do not show any significant shift in the absorption edge this suggests that the organic cation orbitals do not significantly contribute to the valence and conduction bands. However, it has been predicted that the length of organic cation could modify the hole and electron confinement potentials which can significantly influence the carrier transport or confinement in the structure. .4,43 $^{2,4}$

We explored the stability of these $2 \mathrm{D}$ perovskite compounds, for this we prepared the fresh films of $n=14,16,18$ samples and stored them under ambient conditions with relative humidity of $30-50 \%$. To track the changes in the samples, we measured the XRD, absorption and PL emission spectra of these films over 90 days. The XRD results do not show presence of any impurity peaks either from $\mathrm{PbI}_{2}$ or any other related phases, suggesting these samples are quite stable under ambient conditions (Fig. S7, ESI $\dagger$ ). As shown in Fig. 3 a and c the absorption and PL emission spectra of $n=14$ sample show stable absorption edge and PL emission peak positions. The absorption and PL plots of $n=16$ and 18 samples are shown in Fig. S8 and S9 (ESI $\dagger$ ) and these compounds also exhibit quite stable optical properties. 

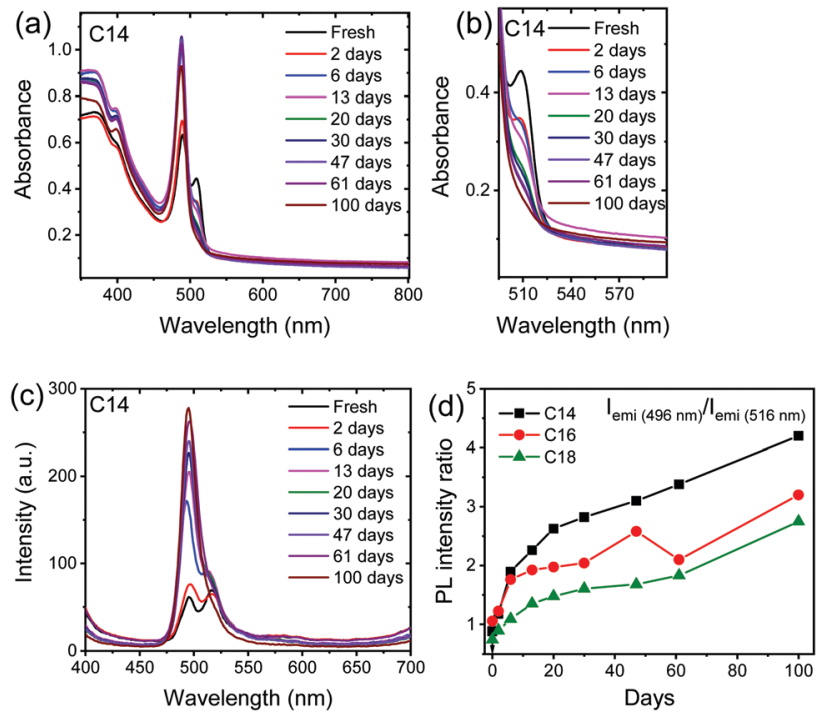

Fig. 3 (a) and (b) Absorption and (c) photoluminescence spectra of thin films of $\left(\mathrm{C}_{14} \mathrm{H}_{29} \mathrm{NH}_{3}\right)_{2} \mathrm{Pbl}_{4} 2 \mathrm{D}$ perovskite thin films stored under ambient conditions. (d) Variation in the photoluminescence intensity ratio of shorter wavelength peak (496 nm) to the longer wavelength peak (516 nm).

The magnified view of the absorption spectra of $n=14$ sample shown in Fig. $3 \mathrm{~b}$ illustrates that the absorption from the HT phase gradually decreases with time suggesting the percentage of HT phase reduces with time and dominant emission from the RT phase was observed, similar trend is observed in the absorption spectra of $n=16$ and 18 samples (Fig. S8, ESI $\dagger$ ).
Moreover, a gradual rise in the PL emission intensity with time was observed in all the 3 samples (Fig. 3c and Fig. S9, ESI $\dagger$ ), which could be related to the passivation of the surface trap states present in the sample either by moisture ${ }^{44}$ or oxygen present in open atmosphere. ${ }^{45}$ The other reason for the increase in PL intensity of peak corresponding to RT phase is conversion of HT phase to RT phase with time. The normalized intensity plots of the fresh samples and aged samples illustrate that the significant portion of the HT phase diminished with time (Fig. S10, ESI $\dagger$ ). Furthermore, the variation in PL intensity ratio of RT phase to HT phase (Fig. 3d) shows a gradual rise in the PL intensity ratio, this also suggest that the HT phase significantly transforms into RT phase after aging. The absorption and PL results leads to a common conclusion that the percentage of HT phase gradually decrease with time.

To check how these 2D perovskite compounds perform in presence of water, we dipped the freshly prepared thin films of $n=14,16$ and 18 in water and monitored the XRD, absorption and PL emission as a function of time. The $n=14$ based films show drastic change in the absorption spectra within 48 hours in water (Fig. 4a). The XRD measurement did not show any diffraction peaks when measured and the substrate was almost transparent which is due to the complete dissolution of perovskite in the water within this period. In comparison to the $n=14$ sample, the $n=16$ and $n=182 \mathrm{D}$ perovskites show stable absorption peak, as shown in Fig. $4 \mathrm{~b}$ and c. The samples immersed in water do not show any impurity peak either from $\mathrm{PbI}_{2}$ or any other hydrated phases illustrating the resistive property of these 2D perovskites towards water (Fig. 4d). However, the XRD
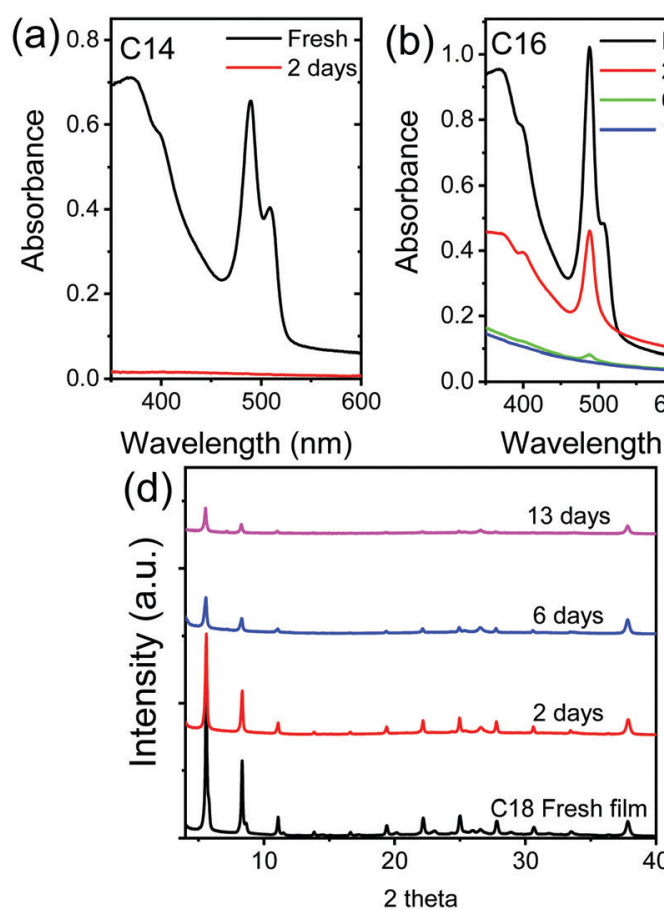
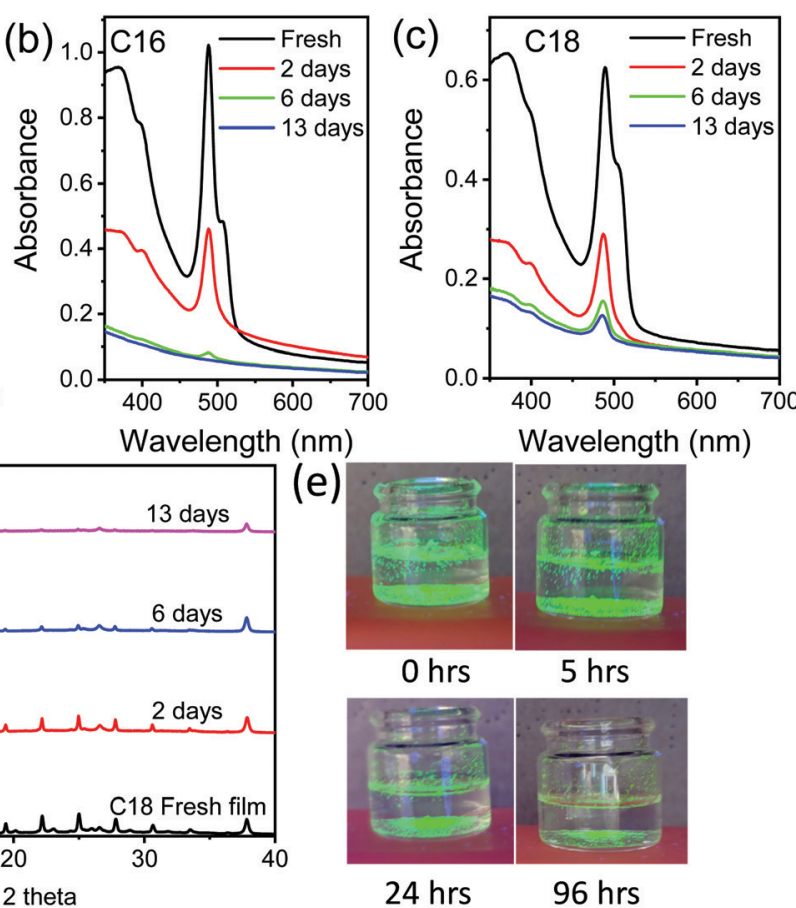

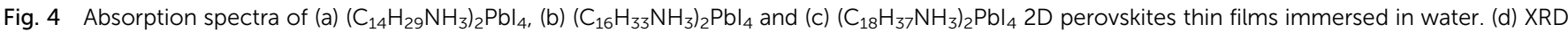

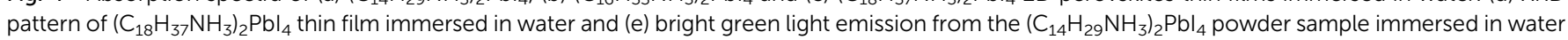
and kept under continuous UV light (365 nm) illumination. 
results show a gradual drop in the intensity of the diffraction peaks, which is due to the partial dissolution of the 2D perovskite compounds in water. The absorption (Fig. 4a-c) and PL emission results (Fig. S11, ESI $\dagger$ ) also supports this claim where a drop in both the absorption and PL emission intensity is observed with time. The effect of water on the phase transition can be clearly seen in Fig. $4 \mathrm{~b}$ and c. The absorption results in Fig. 4 demonstrate major loss of the HT phase compared to the RT phase. The comparison of optical absorption spectra of the samples stored under ambient conditions (Fig. 3 and Fig. S8, ESI $\dagger$ ) and in water (Fig. 4) over a similar time scale illustrates the samples stored in water undergo much faster conversion of HT phase to RT phase. The argument for water affecting the HT phase and transforming it to the RT phase goes as follows. The grain boundaries in the thin films are the most likely place to be attacked by water. In doing so, they will react away and become smaller. When they shrink, they will no longer be in contact with neighboring grains and can rotate more freely and transform back to the RT phase.

The photos of powder samples of $n=14$ dipped in water and under continuous UV light exposure are given in Fig. 4e, the bright green light emission can be observed from the sample which slowly quenches when the samples are kept in water for long time. We further noticed that the spin coated thin-film 2D perovskite samples dissolves much faster in water compared to the bulk powder indicating more resistant nature of powder samples towards water. Note here that the stability results follow a trend of increasing stability with increasing number of carbon atoms in the alkylammonium chain. From these results one could expect a more water-resistant $2 \mathrm{D}$ perovskite with a use of even much longer $(n>18)$ alkylammonium cations so, the future research direction will be focused on the synthesis of other long chain organic cation based 2D perovskites and study their optical properties and stability towards harsh moist conditions.

\section{Conclusion}

In summary, alkylammonium cation based 2D perovskites with varying carbon atoms were successfully synthesized via a simple solution processed method. The powder samples of 2D perovskites show completely reversible phase transitions whereas the thin-films of the same samples possess irreversibility in phase transitions as confirmed from the PL measurements which could be attributed to ease with which the inorganic layers relax upon cooling to room temperature in thin films and powder samples. The absorption and PL measurements show a gradual drop in the percentage of high temperature phase with aging and dominant emission from the RT phase. Furthermore, the presence of moisture helps in achieving the RT phase. Remarkably, the 2D perovskites show a bright green light emission which is stable even in presence of water and the XRD measurement do not show presence of any impurity phase even after water treatment, this property of perovskites is of paramount importance to the design of practical optoelectronic devices.

\section{Conflicts of interest}

There are no conflicts to declare.

\section{Acknowledgements}

We would like to thank Mr Ashutosh Mohanty, IISC Bengaluru for helping us with the DSC measurements. Dr Ilya Sychugov is kindly acknowledged for assisting with absolute quantum yield measurements. B. K. and J. G. gratefully acknowledge the support from the Swedish government through the research initiative "STandUP for ENERGY", the Swedish Foundation for Strategic Research (SSF: RMA15-0130), and the Olle Engqvist Foundation (2017/192).

\section{References}

1 D. Meggiolaro, S. G. Motti, E. Mosconi, A. J. Barker, J. Ball, C. Andrea Riccardo Perini, F. Deschler, A. Petrozza and F. De Angelis, Energy Environ. Sci., 2018, 11, 702-713.

2 R. E. Brandt, V. Stevanović, D. S. Ginley and T. Buonassisi, MRS Commun., 2015, 5, 265-275.

3 Q. Chen, N. De Marco, Y. Yang, T.-B. Song, C.-C. Chen, H. Zhao, Z. Hong, H. Zhou and Y. Yang, Nano Today, 2015, 10, 355-396.

4 D. Shi, V. Adinolfi, R. Comin, M. Yuan, E. Alarousu, A. Buin, Y. Chen, S. Hoogland, A. Rothenberger, K. Katsiev, Y. Losovyj, X. Zhang, P. A. Dowben, O. F. Mohammed, E. H. Sargent and O. M. Bakr, Science, 2015, 347, 519.

5 Q. Dong, Y. Fang, Y. Shao, P. Mulligan, J. Qiu, L. Cao and J. Huang, Science, 2015, 347, 967.

6 NREL, Best Research-Cell Efficiency Chart, https://www.nrel. gov/pv/insights/assets/pdfs/cell-pv-eff-emergingpv.20200406. pdf.

7 Y.-Y. Zhang, S. Chen, P. Xu, H. Xiang, X.-G. Gong, A. Walsh and S.-H. Wei, Chin. Phys. Lett., 2018, 35, 036104.

8 Q. Fu, X. Tang, B. Huang, T. Hu, L. Tan, L. Chen and Y. Chen, Adv. Sci., 2018, 5, 1700387.

9 M. Saliba, T. Matsui, J.-Y. Seo, K. Domanski, J.-P. CorreaBaena, M. K. Nazeeruddin, S. M. Zakeeruddin, W. Tress, A. Abate, A. Hagfeldt and M. Grätzel, Energy Environ. Sci., 2016, 9, 1989-1997.

10 T. Leijtens, K. Bush, R. Cheacharoen, R. Beal, A. Bowring and M. D. McGehee, J. Mater. Chem. A, 2017, 5, 11483-11500.

11 D. P. McMeekin, G. Sadoughi, W. Rehman, G. E. Eperon, M. Saliba, M. T. Hörantner, A. Haghighirad, N. Sakai, L. Korte, B. Rech, M. B. Johnston, L. M. Herz and H. J. Snaith, Science, 2016, 351, 151.

12 L. K. Ono, E. J. Juarez-Perez and Y. Qi, ACS Appl. Mater. Interfaces, 2017, 9, 30197-30246.

13 S. Colella, E. Mosconi, P. Fedeli, A. Listorti, F. Gazza, F. Orlandi, P. Ferro, T. Besagni, A. Rizzo, G. Calestani, G. Gigli, F. De Angelis and R. Mosca, Chem. Mater., 2013, 25, 4613-4618.

14 F. Xu, T. Zhang, G. Li and Y. Zhao, J. Mater. Chem. A, 2017, 5, 11450-11461. 
15 C. Yi, J. Luo, S. Meloni, A. Boziki, N. Ashari-Astani, C. Grätzel, S. M. Zakeeruddin, U. Röthlisberger and M. Grätzel, Energy Environ. Sci., 2016, 9, 656-662.

16 M. C. Brennan, S. Draguta, P. V. Kamat and M. Kuno, ACS Energy Lett., 2018, 3, 204-213.

17 D. J. Slotcavage, H. I. Karunadasa and M. D. McGehee, ACS Energy Lett., 2016, 1, 1199-1205.

18 S. Draguta, O. Sharia, S. J. Yoon, M. C. Brennan, Y. V. Morozov, J. S. Manser, P. V. Kamat, W. F. Schneider and M. Kuno, Nat. Commun., 2017, 8, 200.

19 M. Saliba, T. Matsui, K. Domanski, J.-Y. Seo, A. Ummadisingu, S. M. Zakeeruddin, J.-P. Correa-Baena, W. R. Tress, A. Abate, A. Hagfeldt and M. Grätzel, Science, 2016, 354, 206.

20 Y. Hu, M. F. Aygüler, M. L. Petrus, T. Bein and P. Docampo, ACS Energy Lett., 2017, 2, 2212-2218.

21 B. Kim and S. I. Seok, Energy Environ. Sci., 2020, 13, 805-820.

22 G. Grancini and M. K. Nazeeruddin, Nat. Rev. Mater., 2019, 4, 4-22.

23 W. Fu, H. Liu, X. Shi, L. Zuo, X. Li and A. K. Y. Jen, Adv. Funct. Mater., 2019, 29, 1900221.

24 B. Traore, L. Pedesseau, L. Assam, X. Che, J.-C. Blancon, H. Tsai, W. Nie, C. C. Stoumpos, M. G. Kanatzidis, S. Tretiak, A. D. Mohite, J. Even, M. Kepenekian and C. Katan, ACS Nano, 2018, 12, 3321-3332.

25 J. C. Blancon, A. V. Stier, H. Tsai, W. Nie, C. C. Stoumpos, B. Traoré, L. Pedesseau, M. Kepenekian, F. Katsutani, G. T. Noe, J. Kono, S. Tretiak, S. A. Crooker, C. Katan, M. G. Kanatzidis, J. J. Crochet, J. Even and A. D. Mohite, Nat. Commun., 2018, 9, 2254.

26 C. M. Mauck and W. A. Tisdale, Trends Chem., 2019, 1, 380-393.

27 D. B. Straus and C. R. Kagan, J. Phys. Chem. Lett., 2018, 9, 1434-1447.

28 Z. Wang, Q. Lin, F. P. Chmiel, N. Sakai, L. M. Herz and H. J. Snaith, Nat. Energy, 2017, 2, 17135.

29 Y. Liu, S. Akin, L. Pan, R. Uchida, N. Arora, J. V. Milić, A. Hinderhofer, F. Schreiber, A. R. Uhl, S. M. Zakeeruddin, A. Hagfeldt, M. I. Dar and M. Grätzel, Sci. Adv., 2019, 5, 2543.

30 G. Grancini, C. Roldán-Carmona, I. Zimmermann, E. Mosconi, X. Lee, D. Martineau, S. Narbey, F. Oswald, F. De Angelis, M. Graetzel and M. K. Nazeeruddin, Nat. Commun., 2017, 8, 15684 .
31 M.-H. Li, H.-H. Yeh, Y.-H. Chiang, U. S. Jeng, C.-J. Su, H.-W. Shiu, Y.-J. Hsu, N. Kosugi, T. Ohigashi, Y.-A. Chen, P.-S. Shen, P. Chen and T.-F. Guo, Adv. Mater., 2018, 30, 1801401.

32 C. Ma, C. Leng, Y. Ji, X. Wei, K. Sun, L. Tang, J. Yang, W. Luo, C. Li, Y. Deng, S. Feng, J. Shen, S. Lu, C. Du and H. Shi, Nanoscale, 2016, 8, 18309-18314.

33 K. T. Cho, Y. Zhang, S. Orlandi, M. Cavazzini, I. Zimmermann, A. Lesch, N. Tabet, G. Pozzi, G. Grancini and M. K. Nazeeruddin, Nano Lett., 2018, 18, 5467-5474.

34 Y. Jiang, X. He, T. Liu, N. Zhao, M. Qin, J. Liu, F. Jiang, F. Qin, L. Sun, X. Lu, S. Jin, Z. Xiao, T. Kamiya and Y. Zhou, ACS Energy Lett., 2019, 4, 1216-1224.

35 F. Zhang, D. H. Kim, H. Lu, J.-S. Park, B. W. Larson, J. Hu, L. Gao, C. Xiao, O. G. Reid, X. Chen, Q. Zhao, P. F. Ndione, J. J. Berry, W. You, A. Walsh, M. C. Beard and K. Zhu, J. Am. Chem. Soc., 2019, 141, 5972-5979.

36 D. Thrithamarassery Gangadharan, Y. Han, A. Dubey, X. Gao, B. Sun, Q. Qiao, R. Izquierdo and D. Ma, Sol. $R R L$, 2018, 2, 1700215.

37 S. Kammoun, M. Kamoun, A. Daoud and F. Romain, Phys. Status Solidi A, 1996, 156, 317-329.

38 R. Kind, S. Plesko, H. Arend, R. Blinc, B. Zeks, J. Seliger, B. Lozar, J. Slak, A. Levstik, C. Filipic, V. Zagar, G. Lahajnar, F. Milia and G. Chapuis, J. Chem. Phys., 1979, 71, 2118-2130.

39 M. Koželj, V. Rutar, I. Zupančič, R. Blinc, H. Arend, R. Kind and G. Chapuis, J. Chem. Phys., 1981, 74, 4123-4129.

40 C. Paraggio, V. Salerno, V. Busico and M. Vacatello, Thermochim. Acta, 1980, 42, 185-191.

41 D. G. Billing and A. Lemmerer, New J. Chem., 2008, 32, 1736-1746.

42 S. Barman, N. V. Venkataraman, S. Vasudevan and R. Seshadri, J. Phys. Chem. B, 2003, 107, 1875-1883.

43 M. Safdari, P. H. Svensson, M. T. Hoang, I. Oh, L. Kloo and J. M. Gardner, J. Mater. Chem. A, 2016, 4, 15638-15646.

44 G. E. Eperon, S. N. Habisreutinger, T. Leijtens, B. J. Bruijnaers, J. J. van Franeker, D. W. deQuilettes, S. Pathak, R. J. Sutton, G. Grancini, D. S. Ginger, R. A. J. Janssen, A. Petrozza and H. J. Snaith, ACS Nano, 2015, 9, 9380-9393.

45 S.-C. Liu, Z. Li, Y. Yang, X. Wang, Y.-X. Chen, D.-J. Xue and J.-S. Hu, J. Am. Chem. Soc., 2019, 141, 18075-18082. 